\title{
Variational inequality formulation of circular cone eigenvalue complementarity problems
}

\author{
Xiaoni Chi ${ }^{1}, 2$, Hongjin Wei ${ }^{1}$, Yuqiang Feng ${ }^{3}$ and Jiawei Chen ${ }^{4^{*}}$
}

\section{"Correspondence:}

J.W.Chen713@163.com

${ }^{4}$ School of Mathematics and

Statistics, Southwest University,

Chongqing, 400715, China

Full list of author information is

available at the end of the article

\section{Introduction}

In the past years, the eigenvalue complementarity problems (EiCP) have received great attention, since they have a wide range of applications in engineering, such as the contact problems in mechanics, electrical circuit simulation, dynamic analysis of structural mechanical systems, and vibro-acoustic systems [1-3]. It is noted that the eigenvalue complementarity problem differs from the traditional eigenvalue problem in that the primal and dual variables belong to a closed and convex cone and its dual, respectively, and satisfy a complementarity condition [4]. In [4], the second-order cone eigenvalue complementarity problem (SOCEiCP) was studied via a special variational inequality problem on a compact set defined by a Lorentz cone and a normalization constraint, and moreover, an enumerative algorithm was proposed for solving this problem.

The EiCP where the cone is the nonnegative orthant is called the Pareto eigenvalue complementarity problem. In this case, the spectrum is finite and its cardinality grows exponentially with the size of the problem [5-7]. A number of algorithms have been proposed

(c) 2016 Chi et al. This article is distributed under the terms of the Creative Commons Attribution 4.0 International License (http://creativecommons.org/licenses/by/4.0/), which permits unrestricted use, distribution, and reproduction in any medium, provided you give appropriate credit to the original author(s) and the source, provide a link to the Creative Commons license, and indicate if changes were made. 
for solving EiCP, such as the Path solver [8], the spectral projected gradient algorithm [9], the scaling and projection algorithm [10], and the semismooth Newton method [6].

The second-order cone eigenvalue complementarity problem (SOCEiCP) $[11,12]$ is the EiCP involving the product of second-order cones (or Lorentz cones), which is widely used in game theory, economic equilibrium, engineering, and so on [13-17]. SOCEiCP is regarded as one of the most difficult problems to solve, because the Lorentz spectrum may be the union of finitely many mutually disjoint connected sets and each connected set is either a singleton or a closed interval of positive length (not finite and not countable) [12]. Therefore, it is a difficult task to compute and detect all the eigenvalues of SOCEiCP. A number of semismooth Newton methods have been proposed in [11] for finding a solution of SOCEiCP. A hybrid algorithm which combines a semismooth Newton method with an enumeration algorithm is presented in [4] to detect multiple solutions of SOCE$\mathrm{iCP}$, and it performs very well in practice. A scaling and projection algorithm in [18] is applied to finding a solution of the circular cone eigenvalue complementarity problem (CCEiCP), which is an extension of SOCEiCP.

The circular cone eigenvalue complementarity problem (CCEiCP) [18] consists of finding a real number $\lambda$ and a vector $x \in R^{n} \backslash\{0\}$ such that

$$
\begin{aligned}
& \omega=(\lambda B-A) x, \\
& x \in \mathbf{C}, \quad \omega \in \mathbf{C}^{*}, \\
& x^{T} \omega=0,
\end{aligned}
$$

where $\omega \in R^{n}, A, B \in R^{n \times n}$, and $B$ is positive definite, i.e., $x^{T} B x>0$ for all $x \neq 0$. And $\mathbf{C} \subseteq R^{n}$ is the Cartesian product of circular cones, i.e., $\mathbf{C}=\mathbf{C}_{\theta_{1}}^{n_{1}} \times \mathbf{C}_{\theta_{2}}^{n_{2}} \times \cdots \times \mathbf{C}_{\theta_{m}}^{n_{m}}$, where $n=n_{1}+$ $n_{2}+\cdots+n_{m}$ and $\mathbf{C}_{\theta_{i}}^{n_{i}}$ is the $n_{i}$-dimensional circular cone with $\theta_{i} \in\left(0, \frac{\pi}{2}\right), i=1,2, \ldots, m$. When the rotation angle $\theta_{i}=45^{\circ}$, the circular cone $\mathbf{C}_{\theta_{i}}^{n_{i}}$ reduces to the second-order cone $[19,20]$. Thus CCEiCP includes SOCEiCP as a special case.

Motivated by [4, 18-20], in this paper, we aim to study the variational inequality formulation of $\mathrm{CCEiCP}$, prove the existence of a solution to CCEiCP, and investigate different nonlinear programming formulations of the symmetric and asymmetric CCEiCP, respectively. The different formulations of $\mathrm{CCEiCP}$ provide alternative approaches for finding solutions of CCEiCP, which will play an important role in designing efficient algorithms to find multiple solutions of CCEiCP. We reduce the circular cone eigenvalue complementarity problem (CCEiCP) to a variational inequality problem on a compact convex set. This guarantees that $\mathrm{CCEiCP}$ has at least one solution. Moreover, the symmetric CCEiCP can be reformulated as a nonlinear programming problem $\mathrm{NLP}_{1}$, which maximizes the Rayleigh quotient function on a compact set. Any stationary point of $\mathrm{NLP}_{1}$ is a solution of the symmetric CCEiCP. For the asymmetric CCEiCP, the reduction $\mathrm{NLP}_{1}$ no longer holds. We formulate the asymmetric CCEiCP as another nonlinear programming problem $\mathrm{NLP}_{2}$, and show that any global minimum of $\mathrm{NLP}_{2}$ with an objective function value equal to zero provides a solution of the asymmetric CCEiCP. Moreover, a stationary point of $\mathrm{NLP}_{2}$ is a solution of the asymmetric CCEiCP, if and only if the Lagrange multipliers associated with the equalities in $\mathrm{NLP}_{2}$ are equal to zero. Note that the circular cone $\mathbf{C}_{\theta_{i}}^{n_{i}}$ reduces to the second-order cone, whenever the rotation angle $\theta_{i}=45^{\circ}$. Therefore the presented results in this paper on the symmetric CCEiCP extend that on SOCEiCP in [4]. 
The organization of this paper is as follows. In Section 2, we review some preliminaries including the circular cone, the Euclidean Jordan algebra associated with the secondorder cone, and some concepts. In Section 3, we reduce CCEiCP to a variational inequality problem on a compact convex set, and prove the existence of a solution to CCEiCP. Moreover, we reformulate the symmetric CCEiCP as a nonlinear programming problem $\mathrm{NLP}_{1}$. In Section 4, we formulate the asymmetric CCEiCP as another nonlinear programming problem $\mathrm{NLP}_{2}$, and investigate when a stationary point of $\mathrm{NLP}_{2}$ is a solution of CCEiCP.

In the following, we denote the nonnegative orthant of $R$ by $R_{+}$. We use the symbol $\|\cdot\|$ to denote the Euclidean norm defined by $\|x\|:=\sqrt{x^{T} x}$ for a vector $x$ or the corresponding induced matrix norm. For simplicity, we often use $x=\left(x_{1} ; x_{2: n}\right)$ for the column vector $x=$ $\left(x_{1}, x_{2: n}^{T}\right)^{T}$.

\section{Preliminaries}

In this section, we recall some concepts and results, which include the circular cone [21, $22]$ and the Euclidean Jordan algebra $[13,23]$ associated with the second-order cone (SOC) $K^{n}$.

First, we recall some concepts and the Euclidean Jordan algebra associated with the SOC $[13,23]$. The second-order cone (also called Lorentz cone or ice-cream cone) is defined by

$$
K^{n}=\left\{x=\left(x_{1} ; x_{2: n}\right) \in R \times R^{n-1}:\left\|x_{2: n}\right\| \leq x_{1}\right\}
$$

where $x_{2: n}:=\left(x_{2}, x_{3}, \ldots, x_{n}\right)^{T} \in R^{n-1}$. The Euclidean Jordan algebra for the SOC $K^{n}$ is the algebra defined by

$$
x \circ s=\left(x^{T} s ; x_{1} s_{2: n}+s_{1} x_{2: n}\right), \quad \forall x, s \in R^{n},
$$

with $e=(1,0, \ldots, 0)^{T} \in R^{n}$ being its unit element. Given an element $x=\left(x_{1} ; x_{2: n}\right) \in R \times R^{n-1}$, we define

$$
L(x)=\left(\begin{array}{cc}
x_{1} & x_{2: n}^{T} \\
x_{2: n} & x_{1} I
\end{array}\right)
$$

where $I$ represents the $(n-1) \times(n-1)$ identity matrix. It is easy to verify that $x \circ s=L(x) s$ for any $s \in R^{n}$. Moreover, $L(x)$ is symmetric positive definite (and hence invertible) if and only if $x \in \operatorname{int} K^{n}$.

Now we give some results about the circular cone [21, 22]. The circular cone is a pointed closed convex cone having hyperspherical sections orthogonal to its axis of revolution about which the cone is invariant to rotation. Let its half-aperture angle be $\theta$ with $\theta \in$ $\left(0, \frac{\pi}{2}\right)$. Then the $n$-dimensional circular cone $\mathbf{C}_{\theta}^{n}$ is defined by

$$
\mathbf{C}_{\theta}^{n}:=\left\{x=\left(x_{1} ; x_{2: n}\right) \in R \times R^{n-1}: \cos \theta\|x\| \leq x_{1}\right\}
$$

In fact, it is not difficult to see that

$$
\mathbf{C}_{\theta}^{n}=\left\{x=\left(x_{1} ; x_{2: n}\right) \in R \times R^{n-1}:\left\|x_{2: n}\right\| \leq x_{1} \tan \theta\right\}
$$


Thus the circular cone $\mathbf{C}_{\theta}^{n}$ includes the second-order cone $K^{n}$ as a special case when the half-aperture angle is $\frac{\pi}{4}$. Therefore,

$$
\left[\begin{array}{c}
x_{1} \\
x_{2: n}
\end{array}\right] \in \mathbf{C}_{\theta}^{n} \Leftrightarrow\left[\begin{array}{c}
x_{1} \tan \theta \\
x_{2: n}
\end{array}\right] \in K^{n} \Leftrightarrow\left[\begin{array}{cc}
\tan \theta & 0 \\
0 & I
\end{array}\right]\left[\begin{array}{c}
x_{1} \\
x_{2: n}
\end{array}\right] \in K^{n}
$$

which is equivalent to

$$
\left[\begin{array}{c}
x_{1} \\
x_{2: n}
\end{array}\right] \in \mathbf{C}_{\theta}^{n} \quad \Leftrightarrow \quad T\left[\begin{array}{c}
x_{1} \\
x_{2: n}
\end{array}\right] \in K^{n}
$$

with

$$
T:=\left[\begin{array}{cc}
\tan \theta & 0 \\
0 & I
\end{array}\right] .
$$

It should be pointed out that the matrix $T$ is positive definite and its inverse matrix is

$$
T^{-1}=\left[\begin{array}{cc}
\cot \theta & 0 \\
0 & I
\end{array}\right], \quad \text { where } \cot \theta=\frac{1}{\tan \theta} .
$$

For a convex cone $K$, its dual cone is defined by

$$
K^{*}=\{s:\langle s, x\rangle \geq 0, \forall x \in K\}
$$

It is well known that the second-order cone $K^{n}$ is self-dual, i.e., $\left(K^{n}\right)^{*}=K^{n}$. However, the circular cone is not generally self-dual.

Theorem 2.1 [22] Let $K^{n}$ and $\mathbf{C}_{\theta}^{n}$ be defined as (2) and (3), respectively. Thus $\mathbf{C}_{\frac{\pi}{2}-\theta}^{n}$ is expressed as

$$
\mathbf{C}_{\frac{\pi}{2}-\theta}^{n}=\left\{x=\left(x_{1} ; x_{2: n}\right) \in R \times R^{n-1}:\left\|x_{2: n}\right\| \leq x_{1} \cot \theta\right\} .
$$

Then, we have

(i) $\mathbf{C}_{\theta}^{n}=T^{-1} K^{n}$ and $K^{n}=T \mathbf{C}_{\theta}^{n}$.

(ii) $T K^{n}=\mathbf{C}_{\frac{\pi}{2}-\theta}^{n}$ and $\mathbf{C}_{\frac{\pi}{2}-\theta}^{n}=T^{2} \mathbf{C}_{\theta}^{n}$.

(iii) $\left(\mathbf{C}_{\theta}^{n}\right)^{*}=\mathbf{C}_{\frac{\pi}{2}-\theta}^{n}$ and $\left(\left(\mathbf{C}_{\theta}^{n}\right)^{*}\right)^{*}=\mathbf{C}_{\theta}^{n}$.

\section{A variational inequality formulation of CCEiCP}

In this section, we present a variational inequality formulation of $\mathrm{CCEiCP}$, and prove the existence of a solution to CCEiCP. Moreover, we reformulate the symmetric CCEiCP as a nonlinear programming problem, which will be helpful to detect multiple solutions of the symmetric CCEiCP.

As stated in [24] for EiCP, the CCEiCP can be formulated as the following variational inequality problem $\mathrm{VI}(F, \overline{\mathbf{C}})$ :

Finding a vector $x \in \overline{\mathbf{C}}$ such that

$$
F(x)^{T}(y-x) \geq 0, \quad \forall y \in \overline{\mathbf{C}},
$$


where the function $F: R^{n} \rightarrow R^{n}$ is defined by

$$
F(x)=\left(\frac{x^{T} A x}{x^{T} B x} B-A\right) x,
$$

and $\overline{\mathbf{C}}=\mathbf{C} \cap \Delta$ with

$$
\Delta=\left\{x \in R^{n}: \sum_{i=1}^{m} x_{1}^{i}=1, x_{1}^{i} \tan \theta_{i} \geq 0, i=1,2, \ldots, m\right\} .
$$

\section{Proposition $3.1 \mathrm{VI}(F, \overline{\mathbf{C}})$ has at least one solution.}

Proof Because of the positive definiteness of the matrix $B$, the function $F$ is well defined and continuous on $\overline{\mathbf{C}}$. Furthermore, since $\overline{\mathbf{C}} \subseteq R^{n}$ is compact convex, it follows from Corollary 2.2.5 in [25] that the set of solutions to $\operatorname{VI}(F, \overline{\mathbf{C}})$ is nonempty and compact.

We now set

$$
\widetilde{\mathbf{C}}_{\theta_{i}}^{n_{i}}=\left\{x^{i}=\left(x_{1}^{i} ; x_{2: n_{i}}^{i}\right) \in R \times R^{n_{i}-1}:\left\|x_{2: n_{i}}^{i}\right\|^{2} \leq\left(x_{1}^{i}\right)^{2} \tan ^{2} \theta_{i}\right\}, \quad i=1,2, \ldots, m,
$$

and

$$
\widetilde{\mathbf{C}}=\left(\widetilde{\mathbf{C}}_{\theta_{1}}^{n_{1}} \times \widetilde{\mathbf{C}}_{\theta_{2}}^{n_{2}} \times \cdots \widetilde{\mathbf{C}}_{\theta_{m}}^{n_{m}}\right) \cap \Delta .
$$

It therefore follows that

$$
\overline{\mathbf{C}}=\widetilde{\mathbf{C}} .
$$

Now we present the sufficient optimality conditions for CCEiCP by the variational inequality technique.

Theorem 3.1 If $x \in \overline{\mathbf{C}}$ is a solution of $\mathrm{VI}(F, \overline{\mathbf{C}})$, then $\left(x, \lambda=\frac{x^{T} A x}{x^{T} B x}\right)$ is a solution of CCEiCP.

Proof (i) First of all we consider the case $x_{1}^{i} \tan \theta_{i} \neq 0$ for any $i=1,2, \ldots, m$. From (4) and (9), $x$ is a solution of $\operatorname{VI}(F, \overline{\mathbf{C}})$ if and only if $y=x$ is the optimal solution of the following minimization problem:

$$
\begin{array}{cl}
\min & F(x)^{T} y \\
\text { s.t. } & y \in \widetilde{\mathbf{C}} .
\end{array}
$$

Since the linear independence constraint qualification holds at $x$, we see that $x$ satisfies the following Karush-Kuhn-Tucker (KKT) conditions [26]:

$$
\begin{aligned}
& F(x)=D \alpha+E \beta+\gamma e, \\
& \left\|x_{2: n_{i}}^{i}\right\|^{2} \leq\left(x_{1}^{i}\right)^{2} \tan ^{2} \theta_{i}, \quad i=1,2, \ldots, m, \\
& \alpha_{i}\left(\left\|x_{2: n_{i}}^{i}\right\|^{2}-\left(x_{1}^{i}\right)^{2} \tan ^{2} \theta_{i}\right)=0, \quad i=1,2, \ldots, m,
\end{aligned}
$$




$$
\begin{aligned}
& \alpha_{i} \geq 0, \quad i=1,2, \ldots, m, \\
& x_{1}^{i} \tan \theta_{i} \geq 0, \quad \beta_{i} \geq 0, \quad i=1,2, \ldots, m, \\
& \beta_{i} x_{1}^{i} \tan \theta_{i}=0, \quad i=1,2, \ldots, m, \\
& e^{T} x=1,
\end{aligned}
$$

where

$$
\begin{aligned}
& D=\left[\begin{array}{cccc}
2 x_{1}^{1} \tan ^{2} \theta_{1} & 0 & \cdots & 0 \\
-2 x_{2: n_{1}}^{1} & 0^{1} & \cdots & 0^{1} \\
0 & 2 x_{1}^{2} \tan ^{2} \theta_{2} & \cdots & 0 \\
0^{2} & -2 x_{2: n_{2}}^{2} & \cdots & 0^{2} \\
\vdots & \vdots & \vdots & \vdots \\
0 & 0 & \cdots & 2 x_{1}^{m} \tan ^{2} \theta_{m} \\
0^{m} & 0^{m} & \cdots & -2 x_{2: n_{m}}^{m}
\end{array}\right] \in R^{n \times m} \\
& E=\left[\begin{array}{cccc}
\tan \theta_{1} & 0 & \cdots & 0 \\
0^{1} & 0^{1} & \cdots & 0^{1} \\
0 & \tan \theta_{2} & \cdots & 0 \\
0^{2} & 0^{2} & \cdots & 0^{2} \\
\vdots & \vdots & \vdots & \vdots \\
0 & 0 & \cdots & \tan \theta_{m} \\
0^{m} & 0^{m} & \cdots & 0^{m}
\end{array}\right] \in R^{n \times m}, \quad e=\left[\begin{array}{c}
1 \\
0^{1} \\
1 \\
0^{2} \\
\vdots \\
1 \\
0^{m}
\end{array}\right] \in R^{n}, \\
& \alpha=\left[\begin{array}{c}
\alpha_{1} \\
\alpha_{2} \\
\vdots \\
\alpha_{m}
\end{array}\right] \in R^{m}, \quad \beta=\left[\begin{array}{c}
\beta_{1} \\
\beta_{2} \\
\vdots \\
\beta_{m}
\end{array}\right] \in R^{m} .
\end{aligned}
$$

Here $0^{i}$ is a null vector of dimension $n_{i}-1$, and $\alpha_{i}, \beta_{i}, \gamma$ are the Lagrange multipliers associated with the constraints $\left\|x_{2: n_{i}}^{i}\right\|^{2} \leq\left(x_{1}^{i}\right)^{2} \tan ^{2} \theta_{i}, x_{1}^{i} \tan \theta_{i} \geq 0$, and $e^{T} x=1$ for $i=1,2, \ldots, m$, respectively. On the one hand, it follows from (10), (12), and (15) that

$$
x^{T} F(x)=\gamma .
$$

On the other hand, we have by (5)

$$
x^{T} F(x)=\frac{x^{T} A x}{x^{T} B x} x^{T} B x-x^{T} A x=0 .
$$

Therefore, $\gamma=0$. Then it follows from (5) and (10) that

$$
\omega=\lambda B x-A x, \quad \omega^{T} x=0,
$$

where we let $\lambda=\frac{x^{T} A x}{x^{T} B x}$ and $\omega=D \alpha+E \beta$. Moreover, we have from (10), (12), and (14)

$$
\left\|\omega_{2: n_{i}}^{i}\right\|-\omega_{1}^{i} \cot \theta_{i}=2 \alpha_{i}\left(\left\|x_{2: n_{i}}^{i}\right\|-x_{1}^{i} \tan \theta_{i}\right)-\beta_{i}=-\beta_{i} \leq 0
$$


which implies $\omega^{i} \in \mathbf{C}_{\frac{\pi}{2}-\theta_{i}}^{n_{i}}$ for $i=1,2, \ldots, m$, and therefore $\omega \in \mathbf{C}^{*}$. Hence $(x, \lambda)$ is a solution of CCEiCP.

(ii) Now we consider the case that there exists some $j$ such that $x_{1}^{j} \tan \theta_{j}=0$. Without loss of generality, we assume that there exists only one such $j$ and $j=m$ in the following analysis, since our analysis can easily be extended to the general case. Then $x=\left(u ; x^{m}\right) \in$ $R^{n-n_{m}} \times R^{n_{m}}$ with

$$
u=\left(x^{1} ; x^{2} ; \ldots ; x^{m-1}\right), \quad x^{m}=0
$$

and $x^{i} \neq 0$ for $i=1, \ldots, m-1$. By letting $\lambda=\frac{x^{T} A x}{x^{T} B x}$, we obtain from (1) and (5)

$$
\omega=\left(\begin{array}{c}
v \\
\omega^{m}
\end{array}\right)=F(x)=F\left(\begin{array}{c}
u \\
0
\end{array}\right)=\left[\begin{array}{c}
\lambda B_{11} u-A_{11} u \\
\lambda B_{21} u-A_{21} u
\end{array}\right]=\left[\begin{array}{l}
F_{1}(u) \\
F_{2}(u)
\end{array}\right],
$$

where

$$
\begin{aligned}
\lambda & =\frac{u^{T} A_{11} u}{u^{T} B_{11} u}, \\
A & =\left[\begin{array}{ll}
A_{11} & A_{12} \\
A_{21} & A_{22}
\end{array}\right], \quad B=\left[\begin{array}{ll}
B_{11} & B_{12} \\
B_{21} & B_{22}
\end{array}\right],
\end{aligned}
$$

with $A_{11}, B_{11} \in R^{\left(n-n_{m}\right) \times\left(n-n_{m}\right)}$ and $A_{22}, B_{22} \in R^{n_{m} \times n_{m}}$. Since $x=(u ; 0)$ is a solution of $\mathrm{VI}(F, \overline{\mathbf{C}})$, we get

$$
F(x)^{T}(y-x)=F_{1}(u)^{T}(z-u)+\left(\omega^{m}\right)^{T} y^{m} \geq 0,
$$

where $y=\left(z ; y^{m}\right) \in \overline{\mathbf{C}}$ with $z \in R^{n-n_{m}}$ and $y^{m} \in R^{n_{m}}$. By letting $y^{m}=0$ in (17), we see that $u$ is the solution of the following $\operatorname{VI}\left(F_{1}, \overline{\overline{\mathbf{C}}}\right)$ :

$$
F_{1}(u)^{T}(z-u) \geq 0, \quad \forall z \in \overline{\overline{\mathbf{C}}},
$$

where

$$
\overline{\overline{\mathbf{C}}}=\left(\mathbf{C}_{\theta_{1}}^{n_{1}} \times \cdots \times \mathbf{C}_{\theta_{m-1}}^{n_{m-1}}\right) \cap \bar{\Delta} \subseteq R^{n-n_{m}}
$$

with

$$
\bar{\Delta}=\left\{x \in R^{n-n_{m}}: \sum_{i=1}^{m-1} x_{1}^{i}=1, x_{1}^{i} \tan \theta_{i} \geq 0, i=1,2, \ldots, m-1\right\} .
$$

Then by following the proof of case (i), we can show that $\left(u, \lambda=\frac{u^{T} A_{11} u}{u^{T} B_{11} u}\right)$ is a solution of $\mathrm{CCEiCP}_{1}$ :

$$
\begin{aligned}
& \nu=\lambda B_{11} u-A_{11} u, \\
& \left\|x_{2: n_{i}}^{i}\right\| \leq x_{1}^{i} \tan \theta_{i}, \quad i=1,2, \ldots, m-1,
\end{aligned}
$$




$$
\begin{aligned}
& \left\|\omega_{2: n_{i}}^{i}\right\| \leq \omega_{1}^{i} \cot \theta_{i}, \quad i=1,2, \ldots, m-1, \\
& u^{T} v=0
\end{aligned}
$$

where $u=\left(x^{1} ; x^{2} ; \ldots ; x^{m-1}\right), v=\left(\omega^{1} ; \omega^{2} ; \ldots ; \omega^{m-1}\right) \in R^{n-n_{m}}$.

In order to prove that $\left(x=(u ; 0), \lambda=\frac{u^{T} A_{11} u}{u^{T} B_{11} u}\right)$ is a solution of CCEiCP, in the following we will show

$$
\left\|\omega_{2: n_{m}}^{m}\right\| \leq \omega_{1}^{m} \cot \theta_{m}
$$

where

$$
\omega^{m}=\left(\omega_{1}^{m} ; \omega_{2: n_{m}}^{m}\right)=\lambda B_{21} u-A_{21} u
$$

Since $x=(u ; 0)$ is a solution of $\operatorname{VI}(F, \overline{\mathbf{C}})$ and

$$
F(x)^{T} x=\left(\begin{array}{c}
v \\
\omega^{m}
\end{array}\right)^{T}\left(\begin{array}{l}
u \\
0
\end{array}\right)=v^{T} u=0,
$$

we have

$$
F(x)^{T}(y-x)=F(x)^{T} y \geq 0, \quad \forall y \in \overline{\mathbf{C}},
$$

which is equivalent to

$$
F(x)^{T}(y-x)=F(x)^{T} y \geq 0, \quad \forall y \in \mathbf{C} .
$$

By letting $y=\left(0 ; y^{m}\right) \in R^{n-n_{m}} \times R^{n_{m}}$, we obtain

$$
F(x)^{T} y=\left(\begin{array}{c}
v \\
\omega^{m}
\end{array}\right)^{T}\left(\begin{array}{c}
0 \\
y^{m}
\end{array}\right)=\left(\omega^{m}\right)^{T} y^{m} \geq 0, \quad \forall y^{m} \in \mathbf{C}_{\theta_{m}}^{n_{m}} .
$$

The last relation implies $\omega^{m} \in\left(\mathbf{C}_{\theta_{m}}^{n_{m}}\right)^{*}$, that is, (18) holds.

Combining Proposition 3.1 and Theorem 3.1 yields the following result as regards the existence of a solution of CCEiCP.

\section{Corollary 3.2 CCEiCP has at least one solution.}

From Theorem 3.1, we reduce CCEiCP to $\operatorname{VI}(F, \overline{\mathbf{C}})$. However, the mapping $F$ is not monotone, which precludes CCEiCP from being solved by the efficient algorithms available for variational inequality problems [25]. There is no theoretical guarantee that these algorithms globally converge to a solution of CCEiCP, even if line-search processes are employed [27].

As discussed in $[9,12,28,29]$, if $A$ and $B$ are both symmetric matrices, problem (1) is called the symmetric CCEiCP. As a consequence of Theorem 3.1, we can reformulate the symmetric CCEiCP as the following nonlinear programming problem. 
Theorem 3.3 If $x$ is a stationary point of

$$
\begin{aligned}
\mathrm{NLP}_{1}: \quad \max \quad & f(x)=\frac{x^{T} A x}{x^{T} B x} \\
\text { s.t. } \quad & \left\|x_{2: n_{i}}^{i}\right\|^{2} \leq\left(x_{1}^{i}\right)^{2} \tan ^{2} \theta_{i}, i=1,2, \ldots, m, \\
& \sum_{i=1}^{m} x_{1}^{i}=1, \\
& x_{1}^{i} \tan \theta_{i} \geq 0, \quad i=1,2, \ldots, m,
\end{aligned}
$$

where $A \in R^{n \times n}$ is symmetric and $B \in R^{n \times n}$ is symmetric positive definite, then $\left(x, \lambda=\frac{x^{T} A x}{x^{T} B x}\right)$ is a solution of the symmetric CCEiCP.

Proof Let $f: R^{n} \rightarrow R$ be the Rayleigh quotient function defined by $f(x)=\frac{x^{T} A x}{x^{T} B x}$. Then for any $x \in \widetilde{\mathbf{C}}$ given by (8), the gradient of $f$ at $x$ is

$$
\nabla f(x)=-\frac{2}{x^{T} B x}\left(\frac{x^{T} A x}{x^{T} B x} B x-A x\right) .
$$

Therefore, if $x$ is a stationary point of

$$
\max \{f(x): x \in \widetilde{\mathbf{C}}\}
$$

i.e., $\mathrm{NLP}_{1}$, then it follows from Theorem 3.1 and (9) that $\left(x, \lambda=\frac{x^{T} A x}{x^{T} B x}\right)$ is a solution of the symmetric CCEiCP.

Remark 3.1 (i) Since the feasible set of $\mathrm{NLP}_{1}$ is nonempty and compact, such a stationary point $x^{*}$ always exists. Moreover, the corresponding eigenvalue $\lambda^{*}$ of the symmetric CCEiCP is obtained by $\lambda^{*}=\frac{\left(x^{*}\right)^{T} A x^{*}}{\left(x^{*}\right)^{T} B x^{*}}$.

(ii) $\mathrm{NLP}_{1}$ maximizes the Rayleigh quotient function on a compact set defined by linear constraints and $m$ nonlinear inequalities. Therefore, the symmetric CCEiCP can be efficiently solved by employing the algorithms available [26] or developing new algorithms to compute a stationary point of $\mathrm{NLP}_{1}$.

(iii) The reduction $\mathrm{NLP}_{1}$ only holds in the symmetric CCEiCP. For the asymmetric CCEiCP (where at least one of the matrices $A$ or $B$ is asymmetric), we will formulate another nonlinear programming problem in the next section.

\section{A nonlinear programming formulation of the asymmetric CCEiCP}

In this section, we derive the nonlinear programming problem formulation $\mathrm{NLP}_{2}$ of the asymmetric CCEiCP, and show that any global minimum of $\mathrm{NLP}_{2}$ with an objective function value equal to zero provides a solution of the asymmetric CCEiCP. Moreover, we investigate when a stationary point of $\mathrm{NLP}_{2}$ is a solution of the asymmetric CCEiCP. The nonlinear programming formulation $\mathrm{NLP}_{2}$ of CCEiCP provides an alternative approach for solving CCEiCP, which will play an important role in the design of efficient algorithms for finding multiple solutions to CCEiCP.

Theorem 4.1 CCEiCP has a solution $(x, \omega, \lambda)$ if and only if $(x, \omega, \lambda, y)$ is a global minimum of 


$$
\begin{aligned}
\mathrm{NLP}_{2}: \quad \max \quad & f(x, \omega, \lambda, y)=\|y-\lambda x\|^{2}+\left(x^{T} \omega\right)^{2} \\
\text { s.t. } \quad & \omega-B y+A x=0, \\
& \left\|x_{2: n_{i}}^{i}\right\|^{2} \leq\left(x_{1}^{i}\right)^{2} \tan ^{2} \theta_{i}, \quad i=1,2, \ldots, m, \\
& \left\|\omega_{2: n_{i}}^{i}\right\|^{2} \leq\left(\omega_{1}^{i}\right)^{2} \cot ^{2} \theta_{i}, \quad i=1,2, \ldots, m, \\
& \sum_{i=1}^{m}\left(e^{i}\right)^{T} x^{i}-1=0, \\
& \sum_{i=1}^{m}\left(e^{i}\right)^{T} y^{i}-\lambda=0, \\
& x_{1}^{i} \tan \theta_{i} \geq 0, \quad i=1,2, \ldots, m, \\
& \omega_{1}^{i} \cot \theta_{i} \geq 0, \quad i=1,2, \ldots, m,
\end{aligned}
$$

with $f(x, \omega, \lambda, y)=0$.

Proof Let $y^{i}=\lambda x^{i}, i=1,2, \ldots, m$. It is not difficult to verify that (23) and (24) are equivalent to $\sum_{i=1}^{m} x_{1}^{i}=1$ and $\sum_{i=1}^{m} y_{1}^{i}=\lambda$, respectively. Then we obtain the desired result.

It should be noted that the intersection of the constraints (21), (22), and (25) in NLP 2 represents a convex region due to (9), though the regions defined by the individual constraints (21) and (22) are nonconvex. Thus, the feasible region of $\mathrm{NLP}_{2}$ is convex. Moreover, the functions associated with the constraints (21) and (22) are differentiable everywhere. Therefore, any global minimum of $\mathrm{NLP}_{2}$ is a stationary point which is much easier to compute. Thus it is important to investigate the conditions for such a stationary point to be a solution of the asymmetric CCEiCP.

Theorem 4.2 For any given stationary point $\left(x^{*}, \omega^{*}, \lambda^{*}, y^{*}\right)$ of $N L P_{2},\left(x^{*}, \omega^{*}, \lambda^{*}\right)$ is a solution of CCEiCP if and only if $\delta=\mu=0$, where $\delta$ and $\mu$ are the Lagrange multipliers associated with the equality constraints (23) and (24), respectively.

Proof Let

$$
\begin{aligned}
& \gamma=\left(\begin{array}{c}
\gamma_{1} \\
\gamma_{2} \\
\vdots \\
\gamma_{n}
\end{array}\right) \in R^{n}, \quad \alpha=\left(\begin{array}{c}
\alpha_{1} \\
\alpha_{2} \\
\vdots \\
\alpha_{m}
\end{array}\right) \in R^{m}, \quad \beta=\left(\begin{array}{c}
\beta_{1} \\
\beta_{2} \\
\vdots \\
\beta_{m}
\end{array}\right) \in R^{m}, \\
& \delta \in R, \quad \mu \in R, \quad \zeta=\left(\begin{array}{c}
\zeta_{1} \\
\zeta_{2} \\
\vdots \\
\zeta_{m}
\end{array}\right) \in R^{m}, \quad \eta=\left(\begin{array}{c}
\eta_{1} \\
\eta_{2} \\
\vdots \\
\eta_{m}
\end{array}\right) \in R^{m}
\end{aligned}
$$

be the Lagrange multipliers associated with the constraints (20)-(26), respectively. Then a stationary point $\left(x^{*}, \omega^{*}, \lambda^{*}, y^{*}\right)$ of $\mathrm{NLP}_{2}$ satisfies the following KKT conditions [26]:

$$
2\left(x^{T} \omega\right) \omega-2 \lambda(y-\lambda x)=A^{T} \gamma+D \alpha+E \zeta+\delta e,
$$




$$
\begin{aligned}
& 2\left(x^{T} \omega\right) x=\gamma+F \beta+G \eta, \\
& -2 x^{T}(y-\lambda x)=-\mu, \\
& 2(y-\lambda x)=-B^{T} \gamma+\mu e, \\
& \alpha_{i}\left[\left\|x_{2: n_{i}}^{i}\right\|^{2}-\left(x_{1}^{i}\right)^{2} \tan ^{2} \theta_{i}\right]=0, \quad i=1,2, \ldots, m, \\
& \beta_{i}\left[\left\|\omega_{2: n_{i}}^{i}\right\|^{2}-\left(\omega_{1}^{i}\right)^{2} \cot ^{2} \theta_{i}\right]=0, \quad i=1,2, \ldots, m, \\
& \zeta_{i} x_{1}^{i} \tan \theta_{i}=\eta_{i} \omega_{1}^{i} \cot \theta_{i}=0, \quad i=1,2, \ldots, m, \\
& \alpha_{i} \geq 0, \quad \beta_{i} \geq 0, \quad \zeta_{i} \geq 0, \quad \eta_{i} \geq 0, \quad i=1,2, \ldots, m,
\end{aligned}
$$

where

$$
\begin{aligned}
& D=\left[\begin{array}{cccc}
2 x_{1}^{1} \tan ^{2} \theta_{1} & 0 & \cdots & 0 \\
-2 x_{2: n_{1}}^{1} & 0^{1} & \cdots & 0^{1} \\
0 & 2 x_{1}^{2} \tan ^{2} \theta_{2} & \cdots & 0 \\
0^{2} & -2 x_{2: n_{2}}^{2} & \cdots & 0^{2} \\
\vdots & \vdots & \vdots & \vdots \\
0 & 0 & \cdots & 2 x_{1}^{m} \tan ^{2} \theta_{m} \\
0^{m} & 0^{m} & \cdots & -2 x_{2: n_{m}}^{m}
\end{array}\right] \in R^{n \times m} \\
& F=\left[\begin{array}{cccc}
2 \omega_{1}^{1} \cot ^{2} \theta_{1} & 0 & \cdots & 0 \\
-2 \omega_{2: n_{1}}^{1} & 0^{1} & \cdots & 0^{1} \\
0 & 2 \omega_{1}^{2} \cot ^{2} \theta_{2} & \cdots & 0 \\
0^{2} & -2 \omega_{2: n_{2}}^{2} & \cdots & 0^{2} \\
\vdots & \vdots & \vdots & \vdots \\
0 & 0 & \cdots & 2 \omega_{1}^{m} \cot ^{2} \theta_{m} \\
0^{m} & 0^{m} & \cdots & -2 \omega_{2: n_{m}}^{m}
\end{array}\right] \in R^{n \times m} \\
& E=\left[\begin{array}{cccc}
\tan \theta_{1} & 0 & \cdots & 0 \\
0^{1} & 0^{1} & \cdots & 0^{1} \\
0 & \tan \theta_{2} & \cdots & 0 \\
0^{2} & 0^{2} & \cdots & 0^{2} \\
\vdots & \vdots & \vdots & \vdots \\
0 & 0 & \cdots & \tan \theta_{m} \\
0^{m} & 0^{m} & \cdots & 0^{m}
\end{array}\right] \in R^{n \times m}
\end{aligned}
$$

and

$$
G=\left[\begin{array}{cccc}
\cot \theta_{1} & 0 & \cdots & 0 \\
0^{1} & 0^{1} & \cdots & 0^{1} \\
0 & \cot \theta_{2} & \cdots & 0 \\
0^{2} & 0^{2} & \cdots & 0^{2} \\
\vdots & \vdots & \vdots & \vdots \\
0 & 0 & \cdots & \cot \theta_{m} \\
0^{m} & 0^{m} & \cdots & 0^{m}
\end{array}\right] \in R^{n \times m}
$$


Multiplying (27), (28), and (30) by $x^{T}, \omega^{T}$, and $y^{T}$, respectively, and using (23), (24), and (33), we have

$$
\begin{aligned}
& 2\left(x^{T} \omega\right)^{2}-2 \lambda x^{T}(y-\lambda x)=\gamma^{T} A x+2 \sum_{i=1}^{m} \alpha_{i}\left[-\left\|x_{2: n_{i}}^{i}\right\|^{2}+\left(x_{1}^{i}\right)^{2} \tan ^{2} \theta_{i}\right]+\delta \\
& 2\left(x^{T} \omega\right)^{2}=\gamma^{T} \omega+2 \sum_{i=1}^{m} \beta_{i}\left[-\left\|\omega_{2: n_{i}}^{i}\right\|^{2}+\left(\omega_{1}^{i}\right)^{2} \cot ^{2} \theta_{i}\right] \\
& 2 y^{T}(y-\lambda x)=-\gamma^{T} B y+\mu \lambda .
\end{aligned}
$$

Adding the last three inequalities and using (20), (31), and (32), we obtain

$$
4\left(x^{T} \omega\right)^{2}+2\|y-\lambda x\|^{2}=\delta+\mu \lambda,
$$

i.e., there exists some $(\delta, \mu)$ such that the stationary point $\left(x^{*}, \omega^{*}, \lambda^{*}, y^{*}\right)$ of $\mathrm{NLP}_{2}$ satisfies

$$
2\left(x^{T} \omega\right)^{2}+2 f(x, \omega, \lambda, y)=\delta+\mu \lambda .
$$

(i) Suppose that $\left(x^{*}, \omega^{*}, \lambda^{*}\right)$ is a solution of CCEiCP, i.e., $\left(x^{*}\right)^{T} \omega^{*}=0$ and $\omega^{*}=\lambda^{*} B x^{*}-$ $A x^{*}$. Since $\left(x^{*}, \omega^{*}, \lambda^{*}, y^{*}\right)$ is also a stationary solution of $\mathrm{NLP}_{2}$, we have $\omega^{*}=B y^{*}-A x^{*}$. Then $B y^{*}=\lambda^{*} B x^{*}$ and hence $y^{*}=\lambda^{*} x^{*}$, since $B$ is positive definite. Combining $y^{*}=\lambda^{*} x^{*}$ with $\left(x^{*}\right)^{T} \omega^{*}=0$ yields $f\left(x^{*}, \omega^{*}, \lambda^{*}, y^{*}\right)=0$. Thus we have from (29) $\mu=0$ and therefore by (35) $\delta=0$.

(ii) If $\delta=\mu=0$, we have from (35) $f\left(x^{*}, \omega^{*}, \lambda^{*}, y^{*}\right)=0$, and $\left(x^{*}, \omega^{*}, \lambda^{*}\right)$ is a solution of CCEiCP.

Remark 4.1 Since the constraints (20), (23), (24), (25), and (26) are all linear, the linear independence constraint qualification holds at a stationary point $(x, \omega, \lambda, y)$ of $\mathrm{NLP}_{2}$ if and only if $x_{1}^{i} \tan \theta_{i}>0$ and $\omega_{1}^{i} \cot \theta_{i}>0$ for any $i=1,2, \ldots, m$.

Corollary 4.3 For any given solution $\left(x^{*}, \omega^{*}, \lambda^{*}\right)$ of CCEiCP, there exists a corresponding stationary point $\left(x^{*}, \omega^{*}, \lambda^{*}, y^{*}\right)$ of $N L P_{2}$.

From Theorem 4.2, a stationary point of $\mathrm{NLP}_{2}$ is a solution of CCEiCP if and only if the Lagrange multipliers associated with the equality constraints (23) and (24) in $\mathrm{NLP}_{2}$ are exactly zero. Therefore, the computation of stationary points of $\mathrm{NLP}_{2}$ is a valuable tool for the design of efficient algorithms to find multiple solutions of CCEiCP.

\section{Conclusions}

In this paper, we study the variational inequality formulation of the circular cone eigenvalue complementarity problem $(\mathrm{CCEiCP})$. We reduce $\mathrm{CCEiCP}$ to a variational inequality problem on a compact convex set, which guarantees that CCEiCP has at least one solution. Based on the variational inequality formulation of CCEiCP, the symmetric CCEiCP can be reformulated as a nonlinear programming problem $\mathrm{NLP}_{1}$, whose stationary point is a solution of the symmetric CCEiCP. For the asymmetric CCEiCP, this reduction is no longer valid. We formulate the asymmetric $\mathrm{CCEiCP}$ as another nonlinear programming 
problem $\mathrm{NLP}_{2}$, and show that any global minimum of $\mathrm{NLP}_{2}$ with an objective function value equal to zero provides a solution of the asymmetric CCEiCP. However, contrary to the symmetric case, a stationary point of $\mathrm{NLP}_{2}$ is not sufficient for finding a solution of the asymmetric CCEiCP. Moreover, the sufficient condition is not too strong. The different formulations of CCEiCP provide alternative approaches for solving CCEiCP, which will play an important role in designing efficient algorithms to find multiple solutions of CCEiCP. This is an interesting topic for our future research.

\section{Competing interests}

The authors declare that they have no competing interests.

\section{Authors' contributions}

All authors contributed equally to this paper. They read and approved the final manuscript.

\section{Author details}

'School of Mathematics and Computing Science, Guangxi Colleges and Universities Key Laboratory of Data Analysis and Computation, Guilin University of Electronic Technology, Guilin, 541004, China. ${ }^{2}$ Hubei Province Key Laboratory of Systems Science in Metallurgical Process, Wuhan University of Science and Technology, Wuhan, 430081, China. ${ }^{3}$ College of Science, Wuhan University of Science and Technology, Wuhan, 430081, China. ${ }^{4}$ School of Mathematics and Statistics, Southwest University, Chongqing, 400715, China.

\section{Acknowledgements}

This research is supported by the National Natural Science Foundation of China (Nos. 11401126, 11401487, 71461005), Hubei Provincial Natural Science Foundation (No. 2013CFA131), Hubei Province Key Laboratory of Systems Science in Metallurgical Process (Wuhan University of Science and Technology) (No. Z201401), Guangxi Natural Science Foundation (No. 2014GXNSFFA1 18001), the Fundamental Research Funds for the Central Universities (Nos. SWU113037,

XDJK2014C073), and the Scientific Research Foundation of the Higher Education Institutions of Guangxi (No. ZD2014050), China. The authors are grateful to the editor and the anonymous referees for their valuable comments on this paper.

Received: 28 October 2015 Accepted: 28 February 2016 Published online: 11 March 2016

\section{References}

1. Martins, JAC, Barbarin, S, Raous, M, Pinto da Costa, A: Dynamic stability of finite dimensional linearly elastic systems with unilateral contact and Coulomb friction. Comput. Methods Appl. Mech. Eng. 177, 289-328 (1999)

2. Martins, JAC, Pinto da Costa, A: Stability of finite-dimensional nonlinear elastic systems with unilateral contact and friction. Int. J. Solids Struct. 37, 2519-2564 (2000)

3. Martins, JAC, Pinto da Costa, A, Figueiredo, IN, Júdice, JJ: The directional instability problem in systems with frictional contacts. Comput. Methods Appl. Mech. Eng. 193, 357-384 (2004)

4. Fernandesa, LM, Fukushima, M, Júdice, JJ, Sherali, HD: The second-order cone eigenvalue complementarity problem. Optim. Methods Softw. 31, 24-52 (2016)

5. Adly, S, Rammal, H: A new method for solving eigenvalue complementarity problems. Comput. Optim. Appl. 55, 703-731 (2013)

6. Adly, S, Seeger, A: A nonsmooth algorithm for cone-constrained eigenvalue problems. Comput. Optim. Appl. 49, 299-318 (2011)

7. Seeger, A, Vicente-Perez, J: On cardinality of Pareto spectra. Electron. J. Linear Algebra 22, 758-766 (2011)

8. Dirkse, SP, Ferris, MC: The PATH solver: a non-monotone stabilization scheme for mixed complementarity problems. Optim. Methods Softw. 5, 123-156 (1995)

9. Júdice, JJ, Raydan, M, Rosa, S, Santos, S: On the solution of symmetric eigenvalue complementarity problem by the spectral projected gradient algorithm. Numer. Algorithms 47, 391-407 (2008)

10. Pindo da Costa, A, Seeger, A: Cone-constrained eigenvalue problems: theory and algorithms. Comput. Optim. Appl. 45, 25-57 (2010)

11. Adly, S, Rammal, H: A new method for solving second-order cone eigenvalue complementarity problems. J. Optim. Theory Appl. 165, 563-585 (2015)

12. Seeger, A, Torki, M: On eigenvalues induced by a cone constraint. Linear Algebra Appl. 372, 181-206 (2003)

13. Alizadeh, F, Goldfarb, D: Second-order cone programming. Math. Program. 95, 3-51 (2003)

14. Lobo, M, Vandenberghe, L, Boyd, S, Lebret, H: Applications of second-order cone programming. Linear Algebra Appl. 284, 193-228 (1998)

15. Wang, GQ, Bai, YQ: A new full Nesterov-Todd step primal-dual path-following interior-point algorithm for symmetric optimization. J. Optim. Theory Appl. 154, 966-985 (2012)

16. Che, HT: A smoothing and regularization predictor-corrector method for nonlinear inequalities. J. Inequal. Appl. 2012, 214 (2012)

17. Chen, JW, Liou, YC, Wan, Z, Yao, JC: A proximal point method for a class of monotone equilibrium problems with linear constraints. Oper. Res. Int. J. 15, 275-288 (2015)

18. Pinto Da Costa, A, Seeger, A: Numerical resolution of cone-constrained eigenvalue problems. Comput. Appl. Math. 28, 37-61 (2009)

19. Zhou, JC, Chen, JS, Hung, HF: Circular cone convexity and some inequalities associated with circular cones. J. Inequal. Appl. 2013, 571 (2013) 
20. Bai, YQ, Gao, XR, Wang, GQ: Primal-dual interior-point algorithms for convex quadratic circular cone optimization. Numer. Algebra Control Optim. 5, 211-231 (2015)

21. Dattorro, J: Convex Optimization and Euclidean Distance Geometry. Meboo Publishing, Palo Alto (2005)

22. Zhou, JC, Chen, JS: Properties of circular cone and spectral factorization associated with circular cone. J. Nonlinear Convex Anal. 14, 807-816 (2013)

23. Faraut, U, Korányi, A: Analysis on Symmetric Cones. Oxford University Press, New York (1994)

24. Júdice, JJ, Sherali, H, Ribeiro, IM, Rosa, S: On the asymmetric eigenvalue complementarity problem. Optim. Methods Softw. 24, 549-586 (2009)

25. Facchinei, F, Pang, J: Finite-Dimensional Variational Inequalities and Complementarity Problems. Springer, New York (2003)

26. Bazaraa, MS, Sherali, HD, Shetty, CM: Nonlinear Programming: Theory and Algorithms. Wiley, New York (2006)

27. Brás, C, Fukushima, M, Júdice, J, Rosa, S: Variational inequality formulation of the asymmetric eigenvalue complementarity problem and its solution by means of gap functions. Pac. J. Optim. 8, 197-215 (2012)

28. Queiroz, MG, Júdice, JJ, Humes, JC: The symmetric eigenvalue complementarity problem. Math. Comput. 73, 1849-1863 (2004)

29. Seeger, A, Torki, M: Local minima of quadratic forms on convex cones. J. Glob. Optim. 44, 1-28 (2009)

\section{Submit your manuscript to a SpringerOpen ${ }^{\circ}$ journal and benefit from:}

- Convenient online submission

- Rigorous peer review

- Immediate publication on acceptance

- Open access: articles freely available online

- High visibility within the field

- Retaining the copyright to your article 